\title{
Cluster Analysis for Data Mining and System Identification
}

János Abonyi

Balázs Feil 


\section{Contents}

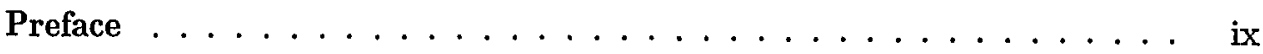

1 Classical Fuzzy Cluster Analysis

1.1 Motivation . . . . . . . . . . . . . . . 1

1.2 Types of Data . . . . . . . . . . . . . . . . . 4

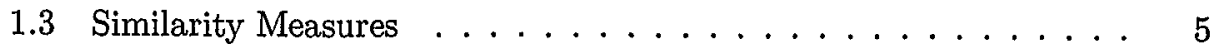

1.4 Clustering Techniques . . . . . . . . . . . . . . 8

1.4.1 Hierarchical Clustering Algorithms . . . . . . . . . . 9

1.4 .2 Partitional Algorithms . . . . . . . . . . . . . . 10

1.5 Fuzzy Clustering . . . . . . . . . . . . . . . . . 17

1.5.1 Fuzzy partition . . . . . . . . . . . . . . 17

1.5.2 The Fuzzy c-Means Functional . . . . . . . . . . . 18

1.5.3 Ways for Realizing Fuzzy Clustering . . . . . . . . . 18

1.5.4 The Fuzzy c-Means Algorithm . . . . . . . . . . . . 19

1.5.5 Inner-Product Norms . . . . . . . . . . . . . . . . . . 24

1.5.6 Gustafson-Kessel Algorithm . . . . . . . . . . . . . . 24

1.5.7 Gath-Geva Clustering Algorithm . . . . . . . . . . . 28

1.6 Cluster Analysis of Correlated Data . . . . . . . . . . . . 32

1.7 Validity Measures. . . . . . . . . . . . . . . . . 40

2 Visualization of the Clustering Results

2.1 Introduction: Motivation and Methods . . . . . . . . . . 47

2.1.1 Principal Component Analysis . . . . . . . . . . . 48

2.1 .2 Sammon Mapping . . . . . . . . . . . . . . 52

2.1.3 Kohonen Self-Organizing Maps . . . . . . . . . . . . . 54

2.2 Fuzzy Sammon Mapping . . . . . . . . . . . . . . . . . . . 59

2.2 .1 Modified Sammon Mapping . . . . . . . . . . . . 60

2.2 .2 Application Examples . . . . . . . . . . . . . 61

2.2 .3 Conclusions . . . . . . . . . . . . . . . 66

2.3 Fuzzy Self-Organizing Map . . . . . . . . . . . . . . 67

2.3.1 Regularized Fuzzy c-Means Clustering . . . . . . . . . 68

2.3 .2 Case Study . . . . . . . . . . . . . . 75

2.3.3 Conclusions . . . . . . . . . . . . . . . . 79 
3 Clustering for Fuzzy Model Identification - Regression

3.1 Introduction to Fuzzy Modelling . . . . . . . . . . . . 81

3.2 Takagi-Sugeno (TS) Fuzzy Models . . . . . . . . . . . . . . 86

3.2.1 Structure of Zero- and First-order TS Fuzzy Models . . . . 87

3.2.2 Related Modelling Paradigms . . . . . . . . . . . . . 92

3.3 TS Fuzzy Models for Nonlinear Regression . . . . . . . . . . . . 96

3.3.1 Fuzzy Model Identification Based on Gath-Geva Clustering . . . . . . . . . . . . . . . 98

3.3.2 Construction of Antecedent Membership Functions . . . . . 100

3.3.3 Modified Gath-Geva Clustering . . . . . . . . . . . . . . 102

3.3.4 Selection of the Antecedent and Consequent Variables . . . 111

3.3.5 Conclusions . . . . . . . . . . . . . . . . . . 115

3.4 Fuzzy Regression Tree . . . . . . . . . . . . . . . . . 115

3.4 .1 Preliminaries . . . . . . . . . . . . . . . . . 120

3.4.2 Identification of Fuzzy Regression Trees based on Clustering Algorithm . . . . . . . . . . . . . . 122

3.4 .3 Conclusions . . . . . . . . . . . . . . . . . . 133

3.5 Clustering for Structure Selection . . . . . . . . . . . . 133

3.5 .1 Introduction . . . . . . . . . . . . . . 133

3.5.2 Input Selection for Discrete Data . . . . . . . . . . . 134

3.5.3 Fuzzy Clustering Approach to Input Selection . . . . . . . 136

3.5.4 Examples . . . . . . . . . . . . . . . . . . . 137

3.5.5 Conclusions . . . . . . . . . . . . . . . . . . . 139

4 Fuzzy Clustering for System Identification

4.1 Data-Driven Modelling of Dynamical Systems . . . . . . . . . . 142

4.1.1 TS Fuzzy Models of SISO and MIMO Systems . . . . . . 148

4.1.2 Clustering for the Identification of MIMO Processes . . . . 153

4.1 .3 Conclusions . . . . . . . . . . . . . . . . . . . 161

4.2 Semi-Mechanistic Fuzzy Models . . . . . . . . . . . . . . . . . . . 162

4.2.1 Introduction to Semi-Mechanistic Modelling . . . . . . . . . 162

4.2.2 Structure of the Semi-Mechanistic Fuzzy Model . . . . . . . 164

4.2.3 Clustering-based Identification of the Semi-Mechanistic Fuzzy Model . . . . . . . . . . . . . 171

4.2 .4 Conclusions . . . . . . . . . . . . . . . . . . . . . . . 182

4.3 Model Order Selection . . . . . . . . . . . . . . . . . . . 183

4.3.1 Introduction . . . . . . . . . . . . . . . . . 183

4.3 .2 FNN Algorithm . . . . . . . . . . . . . . . . . . . 185

4.3.3 Fuzzy Clustering based FNN . . . . . . . . . . . . . . . 187

4.3.4 Cluster Analysis based Direct Model Order Estimation . . . 189

4.3.5 Application Examples . . . . . . . . . . . . . . . . . . . 190

4.3 .6 Conclusions . . . . . . . . . . . . . . . . . . . . . . . . . . 198

4.4 State-Space Reconstruction . . . . . . . . . . . . . . 198

4.4 .1 Introduction . . . . . . . . . . . . . . . . . . . 198 
4.4.2 Clustering-based Approach to

State-space Reconstruction . . . . . . . . . . . . . . 200

4.4.3 Application Examples and Discussion . . . . . . . . . . 208

4.4 .4 Case Study . . . . . . . . . . . . . . . . . 216

4.4 .5 Conclusions . . . . . . . . . . . . . . . . 222

5 Fuzzy Model based Classifiers

5.1 Fuzzy Model Structures for Classification . . . . . . . . . . . 227

5.1.1 Classical Bayes Classifier . . . . . . . . . . . . . . 227

5.1 .2 Classical Fuzzy Classifier . . . . . . . . . . . . . . 228

5.1.3 Bayes Classifier based on Mixture of Density Models . . . . 229

5.1 .4 Extended Fuzzy Classifier . . . . . . . . . . . . . . 229

5.1.5 Fuzzy Decision Tree for Classification . . . . . . . . . . 230

5.2 Iterative Learning of Fuzzy Classifiers . . . . . . . . . . . . . 232

5.2.1 Ensuring Transparency and Accuracy . . . . . . . . . . 233

5.2 .2 Conclusions . . . . . . . . . . . . . . . . . 237

5.3 Supervised Fuzzy Clustering . . . . . . . . . . . . . . . . . . 237

5.3.1 Supervised Fuzzy Clustering - the Algorithm . . . . . . . 239

5.3.2 Performance Evaluation . . . . . . . . . . . . . . . . 240

5.3 .3 Conclusions . . . . . . . . . . . . . . . . . . . 244

5.4 Fuzzy Classification Tree . . . . . . . . . . . . . . . . . 245

5.4.1 Fuzzy Decision Tree Induction . . . . . . . . . . . . 247

5.4.2 Transformation and Merging of the

Membership Functions . . . . . . . . . . . . . . . . 248

5.4 .3 Conclusions . . . . . . . . . . . . . . . . . . 252

6 Segmentation of Multivariate Time-series

6.1 Mining Time-series Data . . . . . . . . . . . . . . . . . . 253

6.2 Time-series Segmentation . . . . . . . . . . . . . . 255

6.3 Fuzzy Cluster based Fuzzy Segmentation . . . . . . . . . . . 261

6.3.1 PCA based Distance Measure . . . . . . . . . . . . . 263

6.3.2 Modified Gath-Geva Clustering for

Time-series Segmentation . . . . . . . . . . . . . 264

6.3.3 Automatic Determination of the Number of Segments . . 266

6.3.4 Number of Principal Components . . . . . . . . . . . 268

6.3.5 The Segmentation Algorithm . . . . . . . . . . . . 269

6.3 .6 Case Studies . . . . . . . . . . . . . . . . . 270

6.4 Conclusions . . . . . . . . . . . . . . . 273

Appendix: Hermite Spline Interpolation . . . . . . . . . . . . . . 275

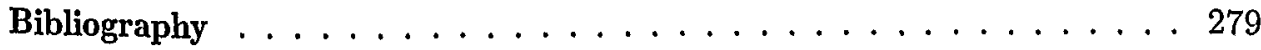

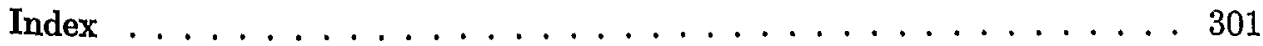

